Sharif University of Technology
Scientia Iranica
Transactions E: Industrial Engineering
http://scientiairanica.sharif.edu
IRAN I CA

\title{
Designing a risk-adjusted CUSUM control chart based on DEA and NSGA-II approaches A case study in healthcare: Cardiovascular patients
}

\author{
N. Rafiei and Sh. Asadzadeh* \\ Department of Industrial Engineering, North Tehran Branch, Islamic Azad University, Tehran, Iran.
}

Received 29 October 2019; received in revised form 24 July 2020; accepted 7 September 2020

\author{
KEYWORDS \\ Control chart design; \\ Risk adjustment; \\ Survival analysis \\ regression models; \\ Non-dominated \\ sorting genetic \\ algorithm (NSGA-II); \\ Data Envelopment \\ Analysis (DEA).
}

\begin{abstract}
Researchers have recently devoted much attention to the development of control charts for monitoring healthcare systems. Accordingly, the purpose of this paper is to design a risk-adjusted cumulative sum (CUSUM) control chart to detect decreasing shifts. The proposed chart is used to monitor the survival times of patients who may be subject to an assignable cause such as human error during a surgery. To this end, risk adjustment is performed to consider the impact of each patient's preoperative risks on survival times using survival analysis regression models. However, using the risk-adjusted CUSUM requires that the chart parameters be determined. Hence, a multi-objective economic-statistical model is proposed and a two-stage solution method composed of Non-dominated Sorting Genetic Algorithm (NSGA-II) and Data Envelopment Analysis (DEA) is implemented to solve the model and obtain the optimal design parameters. The performance of the proposed approach is also studied in a real cardiac surgery center. Finally, to confirm the effectiveness of the proposed multi-objective design, two comparisons with the bi-objective and pure economic designs are made. The results show that the performance of the risk-adjusted CUSUM obtained from the proposed model is better than the two other designs in terms of statistical and economic properties.
\end{abstract}

(C) 2022 Sharif University of Technology. All rights reserved.

\section{Introduction}

Monitoring of hospital and clinical processes has become an important part of modern healthcare systems that enhances the quality of surgical and other treatment services. The most important tools to monitor the effectiveness in the field of healthcare are control charts, which are commonly used to detect shifts in

\footnotetext{
*. Corresponding author.

E-mail addresses: N.rafiei@iua-tnb.ac.ir (N. Rafiei);

Sh_asadzadeh@iua-tnb.ac.ir (Sh. Asadzadeh)
}

doi: $10.24200 /$ sci. 2020.54743 .3895 process parameters [1]. Among them, cumulative sum (CUSUM) control charts are widely applied to statistical monitoring and detecting small shifts in healthcare systems [2]. To this end, four parameters should be determined while making the use of this chart to effectively monitor processes. This includes the sample size, sampling interval, control limits, and the parameter designed for optimal detection of a specific shift. Thus, designing a control chart is defined as the selection of these parameters.

There are several economic and statistical aspects to consider in designing a control chart. The first economic design model was suggested by Duncan [3] to determine the control chart parameters in the pres- 
ence of an assignable cause with a minimum average cost. Duncan's cost model includes the expenses for sampling, out-of-control alarms, detection and repair of assignable causes, and receiving defective products. Another popular model was developed by Lorenzen and Vance [4] which enjoys greater flexibility than Duncan's model. It allows the process to pause or continue throughout the search for detecting and eliminating assignable causes. Since then, a great deal of research was directed at the economic design of control charts. Jiao and Helo [5] suggested an economic design for CUSUM chart based on Taguchi's loss function. Lee [6] studied the design of CUSUM chart for process monitoring with correlated samples. Celano et al. [7] conducted the economic design of CUSUM $t$ chart to monitor short production runs. Fallahnezhad and Golbafian [8] introduced a mathematical model based on the average number of inspected items to form an economic design of the cumulative count of confronting control charts.

However, the economic design of control charts may result in weak statistical features, which makes managers hesitant and dubious about using these charts. Statistical properties include the probability of type-I and type-II errors or the in-control or out-of-control Average Run Length (ARL). To improve the statistical characteristics, Saniga [9] added the probability of type-I and type-II errors as constraints to Duncan's economic model [3] and presented an economic-statistical design. Thus, an economicstatistical design helps gain statistical and economic features simultaneously. Asadzadeh and Khoshalhan [10] proposed a multi-objective model for an economicstatistical design of $\vec{x}$ control charts which, in addition to minimizing an objective function of average expected costs, seeks to maximize the objective functions of test power and in-control Average Run Length (ARL). Niaki et al. [11] compared the economic and economicstatistical designs of MEWMA control chart and suggested a particle swarm optimization method to solve it. Ketabi and Moghadam [12] used a fully adaptive method for model economic and economic-statistical designs of $n p$ control charts in which the cost was developed by Markov Chain approach.

As mentioned earlier, monitoring hospital outputs and clinical processes is considered an essential part of healthcare systems. Patients who are treated in these systems create a heterogeneous population due to their different preoperative features such as age, gender, diabetes, blood pressure, etc. As a result, each patient's mortality after surgery depends on not only surgeon's skills but also unique risk factors before the surgery. Therefore, monitoring and evaluating surgery performance should be adjusted for the effect of patients' risk factors. It should be noted that it is quite impossible to assess the quality of surgery properly without performing the risk-adjustment procedure [2]. Axelrod et al. [13] evaluated the liver and kidney transplantation performance using RiskAdjusted CUSUM (RACUSUM), which detects clinically significant changes in center performance more rapidly. Keefe et al. [14] used a new Bernoulli RACUSUM chart to monitor outputs from cardiac surgery that incorporated outcome information as soon as it would be available. Begun et al. [15] developed a monitoring method based on the RACUSUM chart for early detection of changes in the revision rates after hip replacement. Kim et al. [16] utilized an RACUSUM chart, which monitors the surgical failure, to evaluate the learning curve and establish an appropriate training program for laparoscopic pancreaticoduodenectomy.

Careful investigation of literature reveals that the economic-statistical design of risk-adjusted control charts in healthcare systems has never been addressed. Hence, the present study intends to develop a multiobjective design model for the RACUSUM control chart with economic and statistical considerations. In the proposed model, the design parameters of the RACUSUM chart are determined to minimize the average expected cost as well as the out-of-control average run length $\left(A R L_{1}\right)$, while the in-control average run length $\left(A R L_{0}\right)$ is maximized. There are several methods to solve the suggested model from which Non-dominated Sorting Genetic Algorithm II (NSGA-II) and Data Envelopment Analysis (DEA) are two powerful methods for optimization. NSGA-II is one of the most popular multi-objective evolutionary algorithms due to its capacity to improve the quality of solutions [17]. Therefore, this algorithm is used to determine non-dominated solutions for the suggested model. Then, DEA is applied to find the most efficient solutions. DEA is used to compare and evaluate the relative efficiency of similar units with the same responsibilities [18]. It is widely used to solve multi-criteria problems in economic and management, including the evaluation of hospital services performance and supply chain of a shipping company $[19,20]$.

The rest of the paper is as follows. Section 2 mainly discusses the construction and development of the RACUSUM control chart. Section 3 describes the cost function used in the economic design. Moreover, the multi-objective model for the economicstatistical design of the RACUSUM control chart is developed. Section 4 studies NSGA-II and DEA as solving methods to optimize the model. Section 5 provides a real case study at a cardiac surgery center in Iran to demonstrate the applicability of the proposed procedure in healthcare systems. Section 6 makes two performance comparisons to validate the effectiveness of the proposed multi-objective economic-statistical design. Finally, concluding remarks are given in the last section. 


\section{Monitoring procedure based on risk-adjusted CUSUM control chart}

The challenge of community heterogeneity is apparent in the monitoring of healthcare systems due to the unique preoperative features of patients entering the surgical process. Preoperative features of patients, known as risk factors, include age, gender, diabetes, blood pressure, etc. which affect the monitoring of surgery performance [21]. For instance, patients with more severe risks may experience worse outputs even if they receive the best care. As a result, a patient facing a low surgical risk is much more likely to survive than the one with a high surgical risk. Therefore, surgery performance for each patient depends on not only the surgery quality, but also the unique health record of the patient prior to the operation. Consequently, in order to have an effective monitoring plan, it is necessary to identify the relationship between the mortality rate of a particular disease and its risk factors or to have the monitoring procedure risk-adjusted. Thus, the purpose of the risk-adjusted monitoring scheme is to rapidly detect shifts in patient mortality rates taking into account their risk factors. Given the discrete and continuous outputs, various studies were devoted to developing risk-adjusted control charts. Among the existing methods, a monitoring scheme that is based on patients' survival times is more sensitive to the detection of shifts and changes in the mortality rate than other procedures. It should be noted that the survival time is a variable of reliable nature with some specific features, e.g., being censorable. The censoring mechanism is used to reduce time and cost in surgical processes, which sometimes causes inaccurate recording of survival times. Thus, the reliability of survival times in line with the heterogeneities among patients justifies the use of survival analysis regression models. Accelerated Failure Time (AFT) and Proportional Hazards $(\mathrm{PH})$ are two major regression models in survival analysis which are widely employed to justify heterogeneity in the patients' population and to establish the relationship between survival times and influential covariates (risk factors). The AFT model presents one or more parameters as a function of covariates [22]. The present study makes use of the AFT model for model building and risk adjustment. Accordingly, the parameters of the baseline distribution could be expressed as $\psi_{i}=g\left(\beta, X_{i}\right)$, where $\psi_{i}$ is a vector of distribution parameters and $\beta$ and $X_{i}$ are vectors of regression parameters and covariates, respectively, which reflect the risk factors for the $i$ th patient. Moreover, survival data are often modeled using a member of location-scale and log-location-scale distributions. Weibull is one of the most practical distributions that is known to be helpful in various conditions [23]. As a result, based on the analysis obtained from the real case study, it is assumed that the output variable (survival times) follows Weibull distribution. The probability density and the survival functions of this distribution, denoted by $f$ and $s$, respectively, are as follows:

$$
\begin{aligned}
& f(t)=\frac{\kappa}{\eta}\left(\frac{t}{\eta}\right)^{\kappa-1} \exp \left(-\left(\frac{t}{\eta}\right)^{\kappa}\right), \\
& s(t)=\exp \left(-\left(\frac{t}{\eta}\right)^{\kappa}\right)
\end{aligned}
$$

where $t$ is the recorded value for patients' survival time. Also, $\kappa>0$ and $\eta>0$ are the shape and scale parameters, respectively. Based on the AFT model, if one relates the scale parameter to the unique covariate in the real case study, the probability density and survival functions are restated as:

$$
\begin{aligned}
f(t \mid x)= & \kappa \exp \left(-\left(\beta_{0}+\beta_{1} x_{i}\right)\right)\left(t \exp \left(-\left(\beta_{0}+\beta_{1} x_{i}\right)\right)\right)^{\kappa-1} \\
& \exp \left(-\left(t \exp \left(-\left(\beta_{0}+\beta_{1} x_{i}\right)\right)\right)^{\kappa}\right) \\
s(t \mid x)= & \exp \left(-\left(t \exp \left(-\left(\beta_{0}+\beta_{1} x_{i}\right)\right)\right)^{\kappa}\right),
\end{aligned}
$$

where $\beta_{0}$ and $\beta_{1}$ are the regression model parameters. It should be noted that in the previous equation, the fixed part $\exp \left(\beta_{0}\right)$ has a similar interpretation as $\eta$. As a result, the Weibull distribution has the shape parameter $\kappa$ and the scale parameter $\eta \exp \left(\beta_{1} x_{i}\right)$. Hence, the vector of in-control parameters is defined as $\psi_{i 0}=\left(\kappa, \eta \exp \left(\beta_{1} x_{i}\right)\right)$. It is assumed that $\psi_{i 1}=$ $\left(\kappa, v \eta \exp \left(\beta_{1} x_{i}\right)\right)$ represents the out-of-control condition where $v$ is a predetermined shift such that the CUSUM chart is designed for its optimal detection.

Having defined the relationship between the survival time and preoperative risk factor using the AFT model, it seems essential to design and develop the RACUSUM control chart to monitor survival times. Given the sensitive nature of the healthcare systems with high and irretrievable cost of mistakes with regard to patients' life, there is an attempt to detect decreasing shifts in the mean survival times [24]. As a result, a one-way control chart is suggested and employed for this purpose. The statistic for the risk-adjusted CUSUM chart is calculated as follows:

$$
\begin{aligned}
& Q_{i}=\min \left(0, Q_{i-1}-w_{i}\right), \quad i=1,2, \ldots, \\
& Q_{0}=0
\end{aligned}
$$

where $w_{i}$ is the CUSUM score and is computed as follows:

$$
w_{i}=\log \left\{\frac{L\left(t_{i} \mid \psi_{i 1}\right)}{L\left(t_{i} \mid \psi_{i 0}\right)}\right\},
$$

where $L$ represents the likelihood function. As mentioned earlier, $\psi_{i 0}$ and $\psi_{1 i}$ are the in-control and out- 
of-control nominal values for parameters related to the output variable or survival times.

Thus, it is apparent that the proposed CUSUM chart is designed for rapid detection of shift from $\psi_{i 0}$ to $\psi_{i 1}$. Since a one-way CUSUM chart is used in the present study to detect decreasing shifts in the survival time mean, a lower control limit should be set. In this regard, $L C L$ is the Lower Control Limit and is selected in a way that a desirable in-control ARL is reached. However, to develop the RACUSUM chart, a similar procedure discussed in Sego et al. [21] is implemented to construct the likelihood function in the chart scores. This procedure considers the right-censored mechanism and the patients' survival time is only observed until a pre-determined time or a fixed censored time denoted by $c$. In doing so, the likelihood function is obtained as follows:

$$
L\left(z_{i} \mid \psi_{i}, \delta_{i}\right)=\left[f\left(z_{i}, \psi_{i}\right)\right]^{\delta_{i}}\left[s\left(z_{i}, \psi_{i}\right)\right]^{1-\delta_{i}},
$$

where:

$$
\begin{aligned}
& z_{i}=\min \left(t_{i}, c\right), \\
& \delta_{i}= \begin{cases}1 & \text { if } t_{i} \leq c \\
0 & \text { if } t_{i}>c\end{cases}
\end{aligned}
$$

Substituting the AFT-based probability density and survival functions in Eq. (5), the likelihood function for the RACUSUM chart is obtained. Subsequently, to optimally detect a shift of size $v$ in the survival time mean, the log-likelihood score of the Weibull RACUSUM control chart is given by Eq. (7) shown in Box I. Having obtained the RACUSUM scores, calculation of the control chart statistics is straightforward using Eq. (3). As soon as the updated statistic is less than $L C L$, the RACUSUM control charts trigger a signal.

\section{Economic and economic-statistical designs of the risk-adjusted CUSUM control chart}

The economic design is defined as determining the parameters of an RACUSUM control chart by minimizing a proper cost function. These parameters include $n$ (sample size), $h$ (sampling interval), $L C L$ (lower control limit), and $v$ (coefficient used in the CUSUM for optimal detection of a shift), as discussed earlier. In the present study, the cost function set by Lorenzen and Vance [4] is employed to determine the optimal values of RACUSUM chart parameters. The cost function could be divided into three major parts. The first part includes sampling costs. The second part shows the costs imposed on hospitals (healthcare systems) during the out-of-control surgery conditions. Finally, the last part introduces the cost of detecting and implementing corrective actions for an assignable cause. Moreover, it is assumed that the process begins with an in-control condition and ends when an assignable cause occurs. As the assignable cause happens, the process changes to an out-of-control condition and remains in that situation until it is found and repaired. Then, a new cycle begins. The expected cost in unit time is calculated by dividing the total expected cost during a cycle by the expected length of a cycle. Also, the expected length of a cycle includes four parts: (1) The time that the process remains in control. It is usually assumed that this time is an exponential random variable with mean $\frac{1}{\lambda}$; (2) The time when the process is out of control; (3) The time to detect and identify the assignable cause; and (4) The time for implementing corrective actions to repair the assignable cause. Therefore, by taking all the four parts into account, the expected cycle length equals:

$$
E_{T}=\frac{1}{\lambda}+\left[\left(h \cdot A R L_{1}\right)-\tau\right]+T F+T D .
$$

Subsequently, the total expected cost of a cycle equals:

$$
\begin{aligned}
E_{C}= & A . \frac{\frac{1}{\lambda}+\left[\left(h \cdot A R L_{1}\right)-\tau\right]+\gamma_{1} \cdot T F+\gamma_{2} \cdot T D}{h} \\
& +C O \cdot A R L_{1}+(C F+C D)
\end{aligned}
$$

where the parameters of the above equations are summarized as follows:

- $A R L_{0}:$ In-control average run length;

$$
\begin{aligned}
w_{i}=\log \frac{\left[\frac{\kappa \exp \left(-\beta_{1} x_{i}\right)}{v \eta}\left(\frac{z_{i} \exp \left(-\beta_{1} x_{i}\right)}{v \eta}\right)^{k-1} \exp \left(-\left(\frac{z_{i} \exp \left(-\beta_{1} x_{i}\right)}{v \eta}\right)^{\kappa}\right)\right]^{\delta_{i}}\left[\exp \left(-\left(\frac{z_{i} \exp \left(-\beta_{1} x_{i}\right)}{v \eta}\right)^{\kappa}\right)\right]^{1-\delta_{i}}}{\left[\frac{\kappa \exp \left(-\beta_{1} x_{i}\right)}{\eta}\left(\frac{z_{i} \exp \left(-\beta_{1} x_{i}\right)}{\eta}\right)^{k-1} \exp \left(-\left(\frac{z_{i} \exp \left(-\beta_{1} x_{i}\right)}{\eta}\right)^{\kappa}\right)\right]^{\delta_{i}}\left[\exp \left(-\left(\frac{z_{i} \exp \left(-\beta_{1} x_{i}\right)}{\eta}\right)^{\kappa}\right)\right]^{1-\delta_{i}}} \\
=\left[\left(1-v^{-\kappa}\right)\left(\frac{z_{i} \exp \left(-\beta_{1} x_{i}\right)}{\eta}\right)^{\kappa}\right]-\delta_{i} \kappa \log v .
\end{aligned}
$$


- $A R L_{1}$ : Out-of-control average run length;

- $\tau$ : Expected time of occurrence of the assignable cause between the $j$ th and $j+1$ st samples calculated by the following equation:

$$
\frac{1-(1+\lambda \cdot h) \cdot e^{-\lambda . h}}{\lambda .\left(1-e^{-\lambda . h}\right)},
$$

- TF: The average time required to search for and identify the assignable cause;

- TD: The average time to perform corrective actions;

- A: Sampling costs for each patient;

- CO: Cost imposed on hospital due to out-of-control surgery for each patient because of the occurrence of the assignable cause;

- $C F$ : Cost of searching for and identifying the assignable cause;

- CD: Cost of corrective actions to repair the assignable cause;

- $\gamma_{1}: 1$ if the process does not stop during the search for and investigation into the assignable cause and 0 otherwise;

- $\gamma_{2}$ : 1 if the process does not stop during the repair and elimination of the assignable cause and 0 otherwise.

Finally, the expected cost in unit time imposed on the process is as follows:

$$
E_{A}=\frac{E_{C}}{E_{T}} .
$$

Therefore, the economic design of the RACUSUM chart includes determining the optimal parameters that minimize $E_{A}$. Following Lorenzen and Vance [4], it is practical and helpful to model the costs of designing control charts in accordance with Average Run Length (ARL). It is noteworthy that the present study makes use of simulation method to calculate the values of ARLs.

Next, the main concentration is given to the multi-objective design of the RACUSUM control chart, which is believed to be more beneficial in real practice. The major disadvantage of designing a control chart from the economic point of view is the lack of statistical properties, called the probability of type-I and type-II errors. Therefore, to overcome the statistical weakness of the pure economic design of the RACUSUM control chart, a Multi-Objective Decision Model (MODM) is proposed to consider both the statistical and economic criteria simultaneously. In general, MODM makes use of mathematical programming methods to solve optimization problems with the aim of satisfying several conflicting objective functions at the same time [25]. The multi-objective model of the RACUSUM control chart consists of one economic and two statistical objectives followed by a set of constraints given below:

$$
\begin{aligned}
& \operatorname{Min} E_{A}(D), \\
& \operatorname{Max} \operatorname{ARL}_{0}(D), \\
& \operatorname{Max} 1 / A R L_{1}(D), \\
& \text { s.t. } \\
& E_{A} \leq E_{A}^{U}, \\
& A R L_{0} \geq A R L_{0}^{L}, \\
& A R L_{1} \leq A R L_{1}^{U},
\end{aligned}
$$

where $E_{A}^{U}$ is the desired upper bound on the expected cost. $A R L_{0}^{L}$ and $A R L_{1}^{U}$ are also the pre-determined values showing the lower and upper bounds for $A R L_{0}$ and $A R L_{1}$, respectively. It is remarkable that the MODM model intends to minimize the expected cost for each time unit $\left(E_{A}\right)$, maximize the in-control ARL $\left(A R L_{0}\right)$ to decrease the false alarm rate, and maximize the inverse of out-of-control ARL $\left(1 / A R L_{1}\right)$ to detect out-of-control conditions in a timely manner. Furthermore, $D=(n, h, L C L, v)$ is a possible combination of design parameters that needs to be optimally determined. Therefore, selecting a combination of design parameters for the RACUSUM control chart with optimal objective values is the main goal of the proposed MODM model. The next section elaborates on the solution algorithm based on the integration of Non-dominated Sorting Genetic Algorithm (NSGA-II) and Data Envelopment Analysis (DEA) for optimizing the multi-objective model introduced in Eq. (12).

\section{Solution approach}

To solve the proposed multi-objective economicstatistical model, several algorithms can be proposed. However, simultaneous optimization of multiple objectives creates Pareto solutions. NSGA-II, introduced by Deb et al. [26], is one of the most popular multiobjective evolutionary algorithms for solving a variety of problems which can be used as an efficient method to identify the optimal Pareto set. Several studies dealing with the control chart design, such as the one described in Safaei et al. [17], have implemented NSGA-II to create the optimal Pareto set. Similarly, in this article, NSGA-II is employed to optimally solve the model introduced in Eq. (12). However, there is a challenge while using this algorithm because it often provides many solutions and it is quite difficult to choose the most efficient solution from the optimal Pareto set. To overcome this problem, data envelopment analysis is introduced to rank Pareto solutions and select the 
most efficient ones. Therefore, this study devises a twostep solution method based on NSGA-II and DEA to determine the optimal solutions of the multi-objective economic-statistical model of the RACUSUM control chart. To this end, NSGA-II is shortly summarized and then, the DEA method is introduced.

\subsection{Non-dominated sorting genetic algorithm (NSGA-II)}

NSGA-II makes use of an explicit diversity-preserving mechanism to find Pareto solutions to multi-objective programming problems. In this algorithm, instead of using the offspring populations solely, offspring and parent populations are combined to sort a set of nondominant items. The offspring population is then created out of parent population applying tournament selection, crossover, and mutation operators. The tournament selection operator assumes that each solution $i$ has two attributes in the population, namely a non-dominant rank $\left(r_{i}\right)$ and crowding distance $\left(d_{i}\right)$. Thus, it can be mentioned that solution $i$ wins the competition against solution $j$ if $r_{i}<r_{j}$ or $r_{i}=r_{j}$, and $d_{i}>d_{j}$. This approach ensures better selection from a set of non-dominated solutions [25]. The goal of crossover operator is to exchange information between two parent chromosomes to produce two new offspring for the next population. According to the literature, there are various types of crossover such as one-point, two-point, uniform, and round. In this study, a round crossover operator is applied to produce new chromosomes [27]. This operator is expressed by:

$$
\begin{aligned}
& C h_{1}=\operatorname{round}\left(\operatorname{Par}_{1} \times \text { Alpha }+\operatorname{Par}_{2} \times(1-\text { Alpha })\right), \\
& C h_{2}=\operatorname{round}\left(\operatorname{Par}_{2} \times A l p h a+\operatorname{Par}_{1} \times(1-\text { Alpha })\right),
\end{aligned}
$$

where $\operatorname{Par}_{1}$ and $\mathrm{Par}_{2}$ are the selected parents, respectively. Alpha varies between 0 and 1 , and it has the same dimension as the chromosome matrix. Besides, $C h_{1}$ and $C h_{2}$ are the resulting children. The mutation operator is applied soon after the crossover operator. This operator generates offspring by randomly changing one or several genes in a chromosome. Offspring may thus inherit different characteristics from their parents. Mutation prevents local searching through the search space and increases the probability of finding a global optimum [25].

To find the Pareto optimal set of a multi-objective economic-statistical design model, NSGA-II is defined as follows:

1. Randomly, generate an initial population of size $n$ pop (the number of chromosomes);

2. Compute $A R L_{0}, 1 / A R L_{1}$, and $E_{A}$ for each chromosome;

3. Rank the initial population based on nondominated criteria;

\begin{tabular}{|l|l|l|l|}
\hline$n$ & $h$ & $L C L$ & $v$ \\
\hline
\end{tabular}

Figure 1. A sample chromosome.

4. Compute crowding distance for the initial population;

5. Employ the crossover and mutation operators to generate offspring population of size $n$-pop;

6. Evaluate objectives and constraints for the mentioned offspring population;

7. Combine the two (parent and offspring) populations, rank them, and compute crowding distance;

8. Select a new population of size $n$-pop from the best individuals based on the computed rank and crowding distance;

9. Go to Step 3 and repeat until the termination criterion (number of generations) has been reached.

In this study, $(n, h, L C L, v)$ are the parameters of the RACUSUM control chart which are actually the decision variables of Model (12). Figure 1 shows an example of a chromosome consisting of four genes as design parameters.

It should be noted that the design parameters (decision variables) will be reduced to $(L C L, v)$ due to the described situation in the real case study.

\subsection{Data Envelopment Analysis (DEA)}

DEA is a mathematical programming-based approach which measures the relative efficiency of DecisionMaking Units (DMUs) with multiple inputs and outputs. In this study, to rank the Pareto solutions obtained from NSGA-II, DEA methods are used. For this purpose, non-dominated solutions or design parameters of RACUSUM control chart are considered as DMUs. Also, the two statistical objectives, namely $A R L_{0}$ and $1 / A R L_{1}$, are determined as outputs, and the cost objective $\left(E_{A}\right)$ is the only input of the model. Then, the efficiency of each DMU is calculated using the additive model. Finally, the most efficient DMU is detected through cross-efficiency evaluation technique. These methods are defined briefly in the following subsections.

\subsubsection{Additive model}

The additive model is one of the most important models to determine the efficiency in DEA which is the basis of definition for many other models [25]. One of the main reasons for the importance of this model is that it computes the efficiency completely since it directly attempts to minimize slack variables. However, in other models, such as the CCR and BCC, the detection of slack variables in efficiency is generally performed using another model similar to the additive model at the second stage of efficiency measurement. 
Therefore, one of the advantages of the additive model is that it does not require a two-step approach and the efficiency evaluation of a unit is done by solving a single model. Suppose that there are $m$ DMUs, each with $a$ inputs and $b$ outputs. The values of inputs and outputs for $D M U_{i}(i=1,2, \ldots, m)$ are denoted by $p_{j i}(j=$ $1,2, \ldots, a)$ and $q_{r i}(r=1,2, \ldots, b)$, respectively. The efficiency of a DMU is specified by the additive model expressed as a mathematical programming (Eq. (14)). The mathematical formula for $D M U_{i}$ is given below:

$$
\operatorname{Max} E_{i}(D)=\sum_{r=1}^{b} u_{r} q_{r i}(D)-\sum_{j=1}^{a} e_{j} p_{j i}(D)-T
$$

s.t.

$$
\sum_{r=1}^{b} u_{r} q_{r i}(D)-\sum_{j=1}^{a} e_{j} p_{j i}(D)-T \leq 0
$$

for other design $D$

$$
u_{r}, e_{j} \geq 1
$$

$T$ is free,

where $e_{j}$ and $u_{r}$ are the input and output weights, respectively, and $T$ represents the return to scale. Model (14) should be formulated for each DMU or a combination of design parameters in order to reach a set of weights for maximizing the efficiency of a given DMU. If $E_{i}^{*}=1, D M U_{i}$ is called efficient, while for the case of $E_{i}^{*}<1, D M U_{i}$ is not efficient.

\subsubsection{Cross-efficiency evaluation}

Cross-efficiency evaluation was developed as an extension of DEA to rank efficient DMUs and determine the most efficient one. In a cross-efficiency evaluation, the performance of each efficient DMU is measured according to its optimal weights and the optimal weights of other efficient DMUs [18]. Assuming that the optimal weights of Model (14) for $D M U_{d}$ include $\left(e_{j d}^{*}, u_{r d}^{*}, T_{d}^{*}\right)$, the efficiency of $D M U_{i}(i=1,2, \ldots, m)$ considering the $D M U_{d}$ weights in a peer-evaluated process is calculated as follows:

$$
E_{d i}=\frac{\sum_{r=1}^{b} u_{r d}^{*} q_{r i}}{\sum_{j=1}^{a} e_{j d}^{*} p_{j i}-T_{d}^{*}} .
$$

The mean of all $E_{d i}$ 's is called cross-efficiency and the DMU with the highest cross-efficiency has the best rank. The calculation of the mean is quite straightforward as follows:

$$
\bar{E}_{i}=\frac{\sum_{d=1}^{m} E_{d i}}{m}, \quad i=, 1,2, \ldots, m
$$

Finally, to clarify the application of the RACUSUM control chart in a surgical center using the proposed multi-objective design, Figure 2 is provided to illustrate the summarized steps of the approach.

\section{The case study in the cardiac surgery center}

Cardiovascular disease is a major cause of death around the world and many people die every year because of cardiac diseases. Cardiac surgery is one of the most common surgeries among adults, and given that a person's life depends mainly on his/her heart performance, the sensitivity of this surgery is quite high so that monitoring the patient's survival time after the surgery seems essential. Therefore, the application of the proposed approach is investigated at Imam Ali Cardiac Surgery Center, located in the west of Iran.

For this purpose, a special type of operation called Coronary Artery Bypass Grafting (CABG) surgery was selected, and data on 100 patients including surgery date, surgeon's name, surgery procedure, and survival time were collected. Note that the Parsonnet score is used to determine the preoperative risks for each patient as the only covariate affecting the survival time in the cardiac surgery process [28]. The Parsonnet score is computed based on the sum of various scores given in Table 1.

Once the Parsonnet scores are calculated for each patient, the impact on the survival time should be moderated by the AFT model. Due to the hospital regulations, the survival times of patients who survived during the study were censored on Day 21 . To begin with, the data collected from 100 patients were employed to find an appropriate distribution and estimate the values of in-control parameters. The results revealed that the Parsonnet score data followed gamma distribution with a scale parameter of 5.117 and a shape parameter of approximately 4.208. Then, the Maximum Likelihood Estimation (MLE) was employed to estimate the values of in-control parameters associated with the AFT Weibull model. In doing so, these values were estimated to be $\eta=183744.22, \kappa=$ 1.2066 , and $\beta_{1}=-0.2144$, respectively. Consequently, using Eqs. (2) and (7), the probability density and survival functions of AFT Weibull model in line with the RACUSUM scores can be calculated.

However, the most important part is the deployment of the RACUSUM control chart in the CABG process; thus, it is necessary to determine the four design parameters of the proposed chart. As noted earlier in this study, all patients are monitored individually and sequentially because of the high sensitivity of healthcare systems. Hence, the value of $n$ is constant and is equal to 1 . Furthermore, since patients undergo surgery every four hours at Imam Ali Hospital, the $h$ value is also constant which is equal to 4 . On the other hand, the two other parameters of the RACUSUM chart, namely the coefficient for optimal detection $(v)$ and the lower bound of the control chart $(L C L)$, need to be determined in the process of cardiac surgery so that both statistical and economic properties can be 


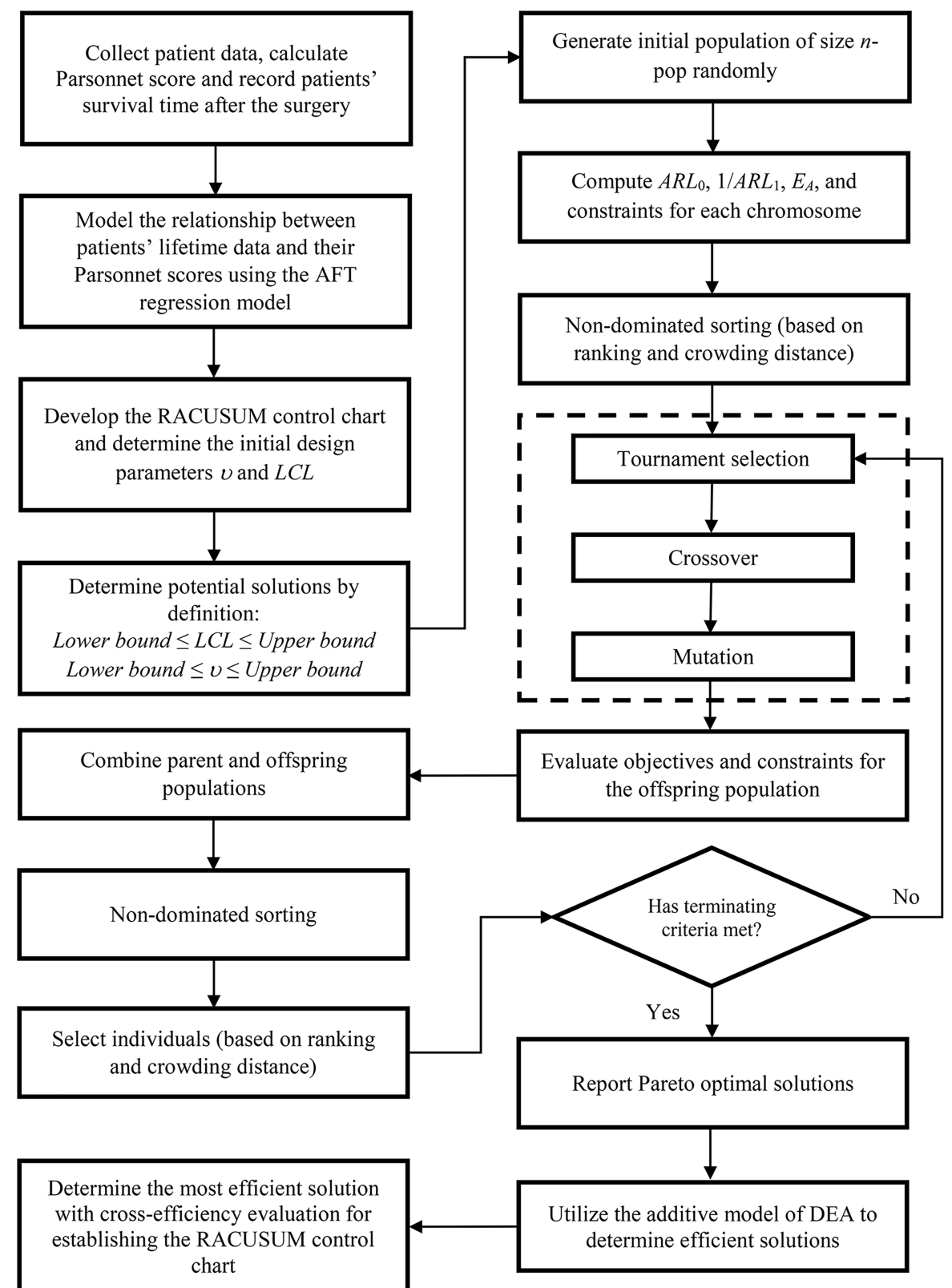

Figure 2. Flowchart of the multi-objective design of RACUSUM control chart at a surgery center.

satisfied. As a result, our proposed MODM model is used to select a combination of $(L C L, v)$ parameters to achieve the desired objectives of minimum expected cost and maximum statistical properties.

In the CABG process, an assignable cause due to the human-resource mistake occurs at $\lambda=001875 h$ rate, thus reducing the patient's survival time by $95 \%$. The sampling cost is 840000 Rials (Iran currency) for each patient because of filling out the Parsonnet questionnaire, carrying out check-ups, and taking actions to obtain Parsonnet score records. In addition, when an assignable cause occurs, the CABG procedure goes to the out-of-control condition. In this case, the cost of check-ups, echocardiography, angiography, surgery, consultant, operation room, consumable products, anesthesia, consumable drugs, ICU beds, nursing services, and public beds is approximately 21623500 Rials, which is imposed on the hospital. The details of the costs imposed on the hospital in the out-of-control condition are reported in Table 2.

In addition, when the CABG process is outof-control, a specialized committee called morbidity or mortality is formed to investigate the root cause of the problem. On average, 4 hours are spent on these actions, and after identifying the root cause of the assignable cause, it takes 2 hours on average to implement corrective actions. An average cost of 16000000 Rials is estimated to find the human-oriented 
Table 1. The preoperative risks for patients used in the calculation of Parsonnet scores.

\begin{tabular}{|c|c|c|c|c|}
\hline \multicolumn{5}{|l|}{ Preoperative risk } \\
\hline Risk factor & & Score & Risk factor & Score \\
\hline \multirow[t]{2}{*}{ Female gender } & & 6 & Left-main disease & 2.5 \\
\hline & $70-75$ & 2.5 & & \\
\hline \multirow[t]{2}{*}{ Age } & $76-79$ & 7 & Morbid obesity & 1 \\
\hline & $80+$ & 11 & & \\
\hline Congestive failure & & 2.5 & preoperative IABP & 4 \\
\hline \multirow{2}{*}{ COPD, severe } & & \multirow{2}{*}{6} & \multirow{2}{*}{ Reoperation } & 10 \\
\hline & & & & 20 \\
\hline Diabetes & & 3 & One valve, aortic & 0 \\
\hline Ejection fraction & $\begin{array}{l}30-49 \% \\
<30 \%\end{array}$ & $\begin{array}{c}6.5 \\
8\end{array}$ & One valve, mitral & 4.5 \\
\hline Hypertension & & 3 & Valve $+\mathrm{ACB}$ & 6 \\
\hline \multicolumn{5}{|l|}{ Special conditions } \\
\hline Cardiac & & Score & Hepato-renal & Score \\
\hline Cardiogenic shock (urinary $<10 \mathrm{cc} / \mathrm{hr}$ ) & & 12 & Cirrhosis & 12.5 \\
\hline Endocarditis, active & & 6.5 & Dialysis dependency & 13.5 \\
\hline Left-ventricular aneurysm resected & & 1.5 & Renal failure, acute or chronic & 3.5 \\
\hline \multicolumn{2}{|l|}{$\begin{array}{l}\text { One valve, tricuspid: } \\
\text { procedure proposed }\end{array}$} & \multicolumn{3}{|l|}{5} \\
\hline \multicolumn{2}{|l|}{ Transmural acute myocardial } & 4 & Vascular & Score \\
\hline \multicolumn{2}{|l|}{ Ventricular septal defect, acute } & 12 & Abdominal aortic aneurysm, asymptomatic & 0.5 \\
\hline \multirow{2}{*}{\multicolumn{2}{|c|}{$\begin{array}{l}\text { Ventricular tachycardia, ventricular } \\
\text { fibrillation, aborted sudden death }\end{array}$}} & \multirow[t]{2}{*}{1} & $\begin{array}{l}\text { Carotid disease (bilateral or } 100 \% \\
\text { unilateral occlusion) }\end{array}$ & 2 \\
\hline & & & Peripheral vascular disease, severe & 3.5 \\
\hline Pulmonary & & Score & & \\
\hline Asthma & & 1 & Miscellaneous & Score \\
\hline Endotracheal tube, preoperative & & 4 & Blood products refused & 11 \\
\hline Idiopathic thrombocytopenic purpura & & 12 & Severe neurologic disorder & 5 \\
\hline \multirow{2}{*}{$\begin{array}{l}\text { Pulmonary hypertension } \\
\text { (mean pressure }>30)\end{array}$} & & 11 & PTCA or catheterization failure & 5.5 \\
\hline & & & Substance abuse & 4.5 \\
\hline \multicolumn{3}{|c|}{ COPD: Chronic Obstructive Pulmonary Disease } & \multicolumn{2}{|l|}{ IABP: Intra-Aortic Balloon Pump } \\
\hline \multicolumn{3}{|c|}{ PTCA: Percutaneous Transluminal Coronary Angioplasty } & ACB: Aortocoronary Bypass & \\
\hline
\end{tabular}

Table 2. The details of the costs imposed on the hospital in the out-of-control condition.

\begin{tabular}{cccc}
\hline Action & Cost (in Rial) & Action & Cost (in Rial) \\
\hline Check-ups & 215000 & Anesthesia & 324000 \\
Echocardiography & 100000 & Consumable products & 2950000 \\
Angiography & 485000 & Consumable drugs & 370000 \\
Consultant & 52000 & ICU bed for two nights & 3811000 \\
Surgery & 1904000 & Nursing services & 809000 \\
Operation room & 483000 & Public bed for eight nights & 10120000 \\
\hline
\end{tabular}


assignable cause and an average cost of 8000000 Rials is spent for corrective actions. Also, the process continues to work while the identification and repair of the assignable cause is being done. Then, according to the estimated parameters with regard to the Parsonnet score and the AFT Weibull model, simulation studies are performed to calculate the statistical indices. It should be noted that in order to minimize the simulation error, the procedure is repeated 10000 times. For each combination of design parameters, the $A R L_{0}$ values are calculated considering no shift in the data, while the $A R L_{1}$ values are recorded when there exists a $95 \%$ reduction in survival time. Moreover, to avoid the high incidence rate of false alarms, to achieve acceptable probability of detection power and to consider the budget constraints using the RACUSUM control chart in the CABG surgery process, a lower bound of 20 , an upper bound of 5 , and an upper bound of 1900000 Rials have been considered for $A R L_{0}, A R L_{1}$, and $E_{A}$, respectively. It is noteworthy that the following limits are applied to the design parameters: $0.01 \leq v \leq 0.2$ and $-1.5 \leq L C L \leq-0.01$. Therefore, the expected cost per time unit associated with the application of the proposed RACUSUM chart to Imam Ali Cardiac Surgery Center is obtained using Eq. (11).

Finally, in order to optimize the multi-objective economic-statistical model of the RACUSUM control chart and to achieve the best possible combination of design parameters, a two-step solution approach is implemented. It should be noted that all calculations related to the solution approach were facilitated under the coded programs in MATLAB (version R2016a) environment. Initially, due to the features of the proposed MODM model and the proper performance of NSGAII, a set of non-dominated solutions was identified using the described algorithm. In other words, the optimal Pareto solutions were determined by implementing NSGA-II with 1000 replications, $n$-pop of size 100, the crossover operator with a probability of 0.2 , and the mutation operator with a probability of 0.9 . The results are reported in Table 3 . Also, the Pareto front for the three objectives $E_{A}, A R L_{0}$, and $1 / A R L_{1}$ is also shown in Figure 3.

After identifying the non-dominated solutions, DEA methods were used to prioritize and select the most effective solution for establishing the RACUSUM control chart at the surgery center. In DEA, any

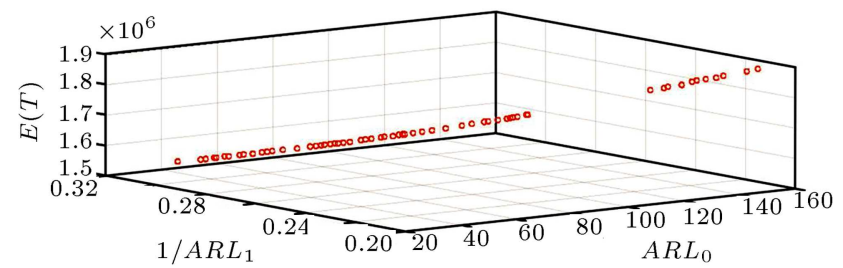

Figure 3. Pareto front for $E_{A}, A R L_{0}$, and $1 / A R L_{1}$.
Table 3. Pareto optimal solutions for the multi-objective economic-statistical design of the RACUSUM control chart.

\begin{tabular}{|c|c|c|c|c|}
\hline \multicolumn{2}{|c|}{ Design parameters } & \multicolumn{3}{|c|}{ Objective function } \\
\hline$v$ & $L C L$ & $\boldsymbol{E}_{A}$ & $A R L_{0}$ & $1 / A R L_{1}$ \\
\hline 0.02 & -1.50 & 1725006.17 & 105.995 & 0.247 \\
\hline 0.05 & -1.44 & 1589433.19 & 68.479 & 0.292 \\
\hline 0.07 & -1.44 & 1557444.81 & 55.752 & 0.305 \\
\hline 0.02 & -1.43 & 1725937.34 & 106.124 & 0.247 \\
\hline 0.12 & -1.42 & 1547031.46 & 49.667 & 0.309 \\
\hline 0.08 & -1.38 & 1555232.69 & 55.118 & 0.306 \\
\hline 0.02 & -1.37 & 1710771.79 & 102.641 & 0.252 \\
\hline 0.02 & -1.35 & 1715010.48 & 103.799 & 0.250 \\
\hline 0.06 & -1.35 & 1571124.64 & 61.068 & 0.299 \\
\hline 0.03 & -1.34 & 1635706.18 & 83.233 & 0.276 \\
\hline 0.05 & -1.34 & 1580663.99 & 66.783 & 0.296 \\
\hline 0.06 & -1.34 & 1567163.32 & 60.558 & 0.301 \\
\hline 0.02 & -1.33 & 1716775.65 & 104.959 & 0.250 \\
\hline 0.03 & -1.33 & 1644061.58 & 84.273 & 0.273 \\
\hline 0.02 & -1.32 & 1713477.95 & 103.062 & 0.251 \\
\hline 0.03 & -1.28 & 1629783.14 & 80.740 & 0.278 \\
\hline 0.02 & -1.24 & 1704920.52 & 101.020 & 0.253 \\
\hline 0.04 & -1.23 & 1593599.92 & 69.038 & 0.291 \\
\hline 0.08 & -1.22 & 1553779.67 & 51.749 & 0.306 \\
\hline 0.06 & -1.20 & 1560712.49 & 57.823 & 0.303 \\
\hline 0.02 & -1.19 & 1698858.61 & 99.045 & 0.255 \\
\hline 0.05 & -1.19 & 1573502.28 & 63.979 & 0.298 \\
\hline 0.03 & -1.17 & 1632935.42 & 81.016 & 0.277 \\
\hline 0.04 & -1.16 & 1597916.06 & 69.616 & 0.289 \\
\hline 0.03 & -1.11 & 1624978.55 & 78.935 & 0.279 \\
\hline 0.01 & -1.07 & 1899125.94 & 150.118 & 0.204 \\
\hline 0.03 & -1.02 & 1622594.21 & 77.414 & 0.280 \\
\hline 0.02 & -1.01 & 1698107.60 & 97.951 & 0.255 \\
\hline 0.03 & -1.01 & 1619648.85 & 76.877 & 0.281 \\
\hline 0.05 & -1.00 & 1566452.67 & 59.089 & 0.301 \\
\hline 0.10 & -0.94 & 1543648.94 & 45.386 & 0.310 \\
\hline 0.02 & -0.92 & 1687088.36 & 96.549 & 0.259 \\
\hline 0.02 & -0.88 & 1681706.96 & 94.539 & 0.260 \\
\hline 0.12 & -0.84 & 1536168.95 & 39.993 & 0.313 \\
\hline 0.03 & -0.83 & 1610764.21 & 75.610 & 0.284 \\
\hline 0.03 & -0.81 & 1605936.12 & 73.015 & 0.286 \\
\hline 0.01 & -0.80 & 1892779.57 & 147.285 & 0.205 \\
\hline 0.01 & -0.70 & 1874373.26 & 142.585 & 0.209 \\
\hline 0.02 & -0.69 & 1665124.77 & 93.358 & 0.266 \\
\hline 0.03 & -0.69 & 1604541.67 & 72.218 & 0.287 \\
\hline 0.02 & -0.68 & 1657098.92 & 90.893 & 0.268 \\
\hline 0.02 & -0.66 & $1,654906.38$ & 88.064 & 0.269 \\
\hline 0.06 & -0.66 & 1552325.62 & 50.880 & 0.307 \\
\hline
\end{tabular}


Table 3. Pareto optimal solutions for the multi-objective economic-statistical design of the RACUSUM control chart (continued).

\begin{tabular}{ccccccc}
\hline \multicolumn{1}{c}{ Design } & parameters & & \multicolumn{3}{c}{ Objective function } \\
\cline { 1 - 2 } $\boldsymbol{y}$ & $\boldsymbol{L} \boldsymbol{C} \boldsymbol{L}$ & & $\boldsymbol{E}_{\boldsymbol{A}}$ & $\boldsymbol{A} \boldsymbol{R} \boldsymbol{L}_{\mathbf{0}}$ & $\mathbf{1} \boldsymbol{A} \boldsymbol{A} \boldsymbol{L}_{\mathbf{1}}$ \\
\cline { 1 - 2 } & -0.63 & & 1609934.16 & 73.659 & 0.285 \\
0.01 & -0.62 & & 1869120.10 & 137.427 & 0.211 \\
0.03 & -0.60 & & 1869355.63 & 140.914 & 0.211 \\
0.06 & -0.54 & & 1598277.20 & 70.672 & 0.289 \\
0.01 & -0.49 & & 1546684.20 & 49.154 & 0.309 \\
0.03 & -0.48 & & $1,864226.13$ & 135.155 & 0.212 \\
0.01 & -0.42 & & 1603011.16 & 71.204 & 0.287 \\
0.01 & -0.41 & & 1841894.75 & 134.194 & 0.217 \\
0.02 & -0.34 & & 1650667.91 & 86.008 & 0.271 \\
0.06 & -0.25 & & 1544854.32 & 46.867 & 0.310 \\
0.01 & -0.22 & & 1836949.32 & 130.496 & 0.218 \\
0.02 & -0.16 & & 1639932.48 & 84.086 & 0.274 \\
0.02 & -0.14 & & 1644105.69 & 84.916 & 0.273 \\
0.01 & -0.09 & & 1832831.55 & 129.904 & 0.219 \\
0.01 & -0.03 & & 1827862.32 & 126.074 & 0.220 \\
\hline
\end{tabular}

combination of design parameters, namely $(L C L, v)$, is considered a DMU. Since DEA methods select the most efficient DMU with the minimum input value and maximum output value, the cost function was considered as the only input while the statistical properties were the two outputs. The additive model was then used to identify the efficient DMUs. Based on the results of the additive model, 10 DMUs were selected as a combination of efficient design parameters. Finally, these 10 DMUs were considered as the input data for the cross-efficiency evaluation technique and the most efficient DMU was detected. The results are shown in Table 4.

From Table 4, it is remarkable that the crossefficiency evaluation technique offers $v=0.08$ and
$L C L=-1.38$ as the most efficient combination of design parameters for the RACUSUM control chart with the best economic and statistical properties $\left(E_{A}=\right.$ 1555232.69 Rials, $A R L_{0}=55.118$, and $1 / A R L_{1}=$ $0.306)$.

\section{Performance comparison}

In this section, the performance of the proposed multiobjective economic-statistical design model is compared with those of the bi-objective statistical design and the pure economic design model to investigate its effectiveness. The bi-objective model is similar to the multi-objective model, presented by Eq. (12) in Section 3 , with a difference that the expected cost for each time unit $\left(E_{A}\right)$ is omitted. Therefore, this model can be introduced with two statistical objectives, $A R L_{0}$ and $1 / A R L_{1}$, which is rewritten with a set of constraints as follows:

$$
\begin{aligned}
& \operatorname{Max} A R L_{0}(D), \\
& \operatorname{Max} 1 / A R L_{1}(D), \\
& \text { s.t. } \\
& E_{A} \leq E_{A}^{U}, \\
& A R L_{0} \geq A R L_{0}^{L}, \\
& A R L_{1} \leq A R L_{1}^{U} .
\end{aligned}
$$

To compare this model with the multi-objective one,

\begin{tabular}{|c|c|c|c|c|c|c|}
\hline \multirow{2}{*}{$\begin{array}{c}\text { DMUs } \\
(L C L, v)\end{array}$} & \multirow{2}{*}{$\begin{array}{c}\begin{array}{c}\text { Optimal input } \\
\text { weight }\end{array} \\
e_{1}^{*}\end{array}$} & \multicolumn{2}{|c|}{$\begin{array}{l}\text { Optimal output } \\
\text { weight }\end{array}$} & \multirow{2}{*}{$\begin{array}{c}\text { Optimal } \\
\text { weight }\end{array}$} & \multirow{2}{*}{$\begin{array}{c}\text { Efficiency } \\
\text { Additive } \\
\text { model }\end{array}$} & \multirow{2}{*}{$\begin{array}{l}\text { Cross- } \\
\text { efficiency }\end{array}$} \\
\hline & & $u_{1}^{*}$ & $u_{2}^{*}$ & & & \\
\hline$(-1.42,0.12)$ & 1.29 & 1685.837 & 125360.81 & 1872173.26 & 1 & 0.93 \\
\hline$(-1.38,0.08)$ & 1.20 & 1858.815 & 37505.95 & 1752593.07 & 1 & 0.94 \\
\hline$(-1.34,0.05)$ & 1.66 & 5683.617 & 390072.56 & 2129647.87 & 1 & 0.91 \\
\hline$(-1.20,0.06)$ & 1.00 & 4090.747 & 2538116.05 & 554369.47 & 1 & 0.93 \\
\hline$(-1.19,0.05)$ & 1.49 & 4099.163 & 319192.26 & 1980022.60 & 1 & 0.92 \\
\hline$(-1.07,0.01)$ & 1.62 & 7658.709 & 902715.92 & 1740017.21 & 1 & 0.70 \\
\hline$(-0.84,0.12)$ & 1.15 & 420.5552 & 44140.31 & 1741218.29 & 1 & 0.90 \\
\hline$(-0.69,0.02)$ & 1.70 & 6153.724 & 343148.33 & 2165886.81 & 1 & 0.82 \\
\hline$(-0.68,0.02)$ & 1.66 & 5626.94 & 297469.39 & 2167488.56 & 1 & 0.83 \\
\hline$(-0.49,0.06)$ & 1.00 & 1122.886 & 1.00 & 1491261.38 & 1 & 0.93 \\
\hline
\end{tabular}
its application to the cardiac surgery center has been studied. According to the characteristics of the biobjective model, NSGA-II was applied for realizing non-dominated solutions and the Pareto front was

Table 4. Efficient solutions obtained from the DEA additive model and cross-efficiency evaluation technique. 
Table 5. Comparison of the multi-objective and bi-objective designs.

\begin{tabular}{|c|c|c|c|c|}
\hline Design & $(L C L, v)$ & $A R L_{0}$ & $A R L_{1}$ & $E_{A}$ \\
\hline Multi-objective design & $(-1.38,0.08)$ & 55.118 & 3.268 & 1555232.69 \\
\hline Bi-objective design & $(-0.69,0.02)$ & 92.358 & 3.763 & 1665124.77 \\
\hline
\end{tabular}

Table 6. Comparison of the multi-objective and pure economic design.

\begin{tabular}{|c|c|c|c|c|}
\hline Design & $(\boldsymbol{L} C L, v)$ & $A R L_{0}$ & $A R L_{1}$ & $E_{A}$ \\
\hline Multi-objective design & $(-1.38,0.08)$ & 55.118 & 3.268 & 1555232.69 \\
\hline Economic design & $(-0.17,0.18)$ & 28.097 & 3.185 & 1534632.37 \\
\hline
\end{tabular}

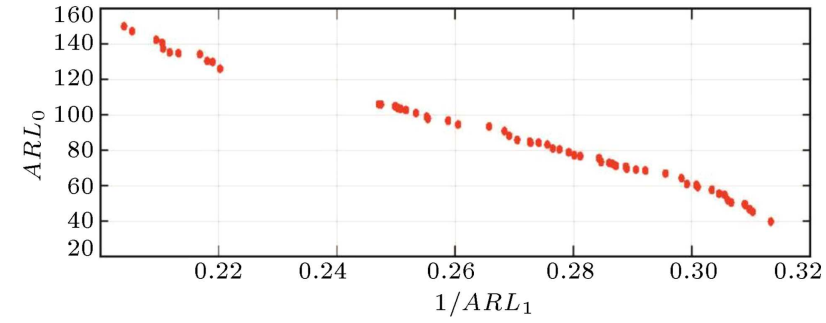

Figure 4. Pareto front for $A R L_{0}$ and $1 / A R L_{1}$.

determined using this algorithm. The Pareto front for $A R L_{0}$ and $1 / A R L_{1}$ of the bi-objective design is shown in Figure 4.

Upon recognizing the non-dominated solutions, $v=0.02$ and $L C L=-0.69$ were selected as the best combination of bi-objective design parameters for the RACUSUM control chart. The optimal values corresponding to multi-objective and bi-objective designs are given in Table 5 .

Table 5 indicates that the bi-objective design model increases the value of $A R L_{0}$ effectively, while the multi-objective design has better performance with regard to $A R L_{1}$ and $E_{A}$. The bi-objective design managed to increase the $A R L_{0}$ by $69.3 \%$. However, $A R L_{1}$ and $E_{A}$ values have been negatively raised by $15.2 \%$ and $7.1 \%$, respectively. Thus, the results confirm that the multi-objective design outperforms the biobjective design in terms of detection power and the expected cost.

Finally, the performance of the multi-objective design is compared with that of the pure economic design model. Table 6 depicts the optimal parameters of multi-objective design and pure economic design. It is apparent that as $E_{A}$ increases by $1.3 \%$ in the multiobjective design compared to the economic design, the $A R L_{0}$ of the multi-objective design increases by $96 \%$ as well. However, no significant difference is observed for $A R L_{1}$ in both designs. Therefore, the comparisons revealed that the $A R L_{0}$ increased dramatically with a slight increase in the cost. Consequently, the multiobjective design could be effectively applied with a significant improvement in statistical properties of the RACUSUM control chart.

\section{Conclusion}

Considering the importance of healthcare systems, this paper proposed a multi-objective economicstatistical model to design the Risk-Adjusted CUSUM (RACUSUM) control chart to effectively monitor patients' lifetime. First, the RACUSUM chart was devised based on a class of survival analysis regression models called the Accelerated Failure Time (AFT) model taking the preoperative risks of each patient into account. It was assumed that the cardiac surgery process in a hospital was affected by an assignable cause resulting from the human mistakes, which caused a decrease in the survival time of patients. Thus, a multi-objective economic-statistical design model was addressed to determine the parameters of RACUSUM chart so that both the economic and statistical properties could be met simultaneously. Due to the constant sample size and sampling interval, while applying the RACUSUM chart to the healthcare system, the control chart design parameters were considered to be the lower control limit and the coefficient for optimal shift detection denoted by $L C L$ and $v$, respectively. In order to determine the optimal values of these parameters, a two-stage solution algorithm was employed. The NSGA-II was used in order to obtain the optimal Pareto set taken from design parameters, and the DEA methods were implemented to rank the solutions and choose the most efficient one. It should be noted that each combination of design parameters was considered as a DMU, and as the efficient DMUs were determined using the additive model, a cross-efficiency evaluation method was employed to select the final solution. Finally, the application of the proposed multi-objective model and the proposed solution method was described at a real cardiac surgery center (hospital) located in the west of Iran. Furthermore, two comparisons were performed with the bi-objective and pure economic design models. The results clearly revealed that the performance of the multi-objective design was relatively superior to the bi-objective design. Likewise, in comparison with pure economic design, the multi-objective design offers better statistical properties although it slightly 
increases the imposed costs. As a result, in general, the proposed approach to designing the RACUSUM control charts can be effectively applied taking the economic and statistical properties into account while monitoring the survival times of patients in healthcare systems. An interesting area worthy of continued research efforts includes the multi-objective design of the RACUSUM control charts in the presence of multiple assignable causes.

\section{Acknowledgements}

The authors express their appreciation of and gratitude for the support of the Vice Chancellor for Research and Technology of Kermanshah University of Medical Sciences.

\section{References}

1. Woodall, W.H., Fogel, S.L., and Steiner, S.H. "The monitoring and improvement of surgical-outcome quality", Journal of Quality Technology, 47(4), pp. 383399 (2015).

2. Rasmussen, T.B, Ulrichsen, S.P., and N $\phi$ rgaard, M. "Use of risk-adjusted CUSUM charts to monitor 30day mortality in Danish hospital", Clinical Epidemiology, 10, pp. 445-456 (2018).

3. Duncan, A.J. "The economic design of $\bar{X}$ charts used to maintain current control of a process", Journal of the American Statistical Association, 51(274), pp. 228-242 (1956).

4. Lorenzen, T.J. and Vance, L.C. "The economic design of control charts: a unified approach", Technometrics, 28(1), pp. 3-10 (1986).

5. Jiao, J.R. and Helo, P.T. "Optimization design of a CUSUM control chart based on Taguchi's loss function", The International Journal of Advanced Manufacturing Technology, 35(11-12), pp. 1234-1243 (2008).

6. Lee, M.H. "Economic design of cumulative sum control charts for monitoring a process with correlated samples", Communications in Statistics-Simulation and Computation, 39(10), pp. 1909-1922 (2010).

7. Celano, G., Castagliola, P., and Trovato, E. "The economic performance of a CUSUM $t$ control chart for monitoring short production runs", Quality Technology \& Quantitative Management, 9(4), pp. 329-354 (2012).

8. Fallahnezhad, M.S. and Golbafian, V. "Economic design of cumulative count of conforming control charts based on average number of inspected items", Scientia Iranica, Transactions E, Industrial Engineering, 24(1), pp. 330-341 (2017).

9. Saniga, E.M. "Economic statistical control chart designs with an application to $\bar{X}$ and R charts", Technometrics, 31(3), pp. 313-320 (1989).
10. Asadzadeh, S. and Khoshalhan, F. "Multiple-objective design of an $\bar{X}$ control chart with multiple assignable causes", The International Journal of Advanced Manufacturing Technology, 43(3-4), pp. 312-322 (2009).

11. Niaki, S.T.A., Malaki, M., and Ershadi, M.J. "A particle swarm optimization approach on economic and economic-statistical designs of MEWMA control charts", Scientia Iranica, Transactions E, Industrial Engineering, 18(6), pp. 1529-1536 (2011).

12. Katebi, M. and Moghadam, M.B. "Optimal statistical, economic and economic statistical designs of attribute np control charts using a full adaptive approach", Communications in Statistics-Theory and Methods, 48(18), pp. 4528-4549 (2019).

13. Axelrod, D.A., Kalbfleisch, J.D., Sun, R.J., et al. "Innovations in the assessment of transplant center performance: implications for quality improvement", American Journal of Transplantation, 9(4p2), pp. 95969 (2009).

14. Keefe, M.J., Loda, J.B., Elhabashy, A.E., et al. "Improved implementation of the risk-adjusted Bernoulli CUSUM chart to monitor surgical outcome quality", International Journal for Quality in Health Care, 29(3), pp. 343-348 (2017).

15. Begun, A., Kulinskaya, E., and MacGregor, A.J. "Risk-adjusted CUSUM control charts for shared frailty survival models with application to hip replacement outcomes: a study using the NJR dataset", $B M C$ Medical Research Methodology, 19(1), pp. 1-15 (2019).

16. Kim, S., Yoon, Y., Han, H., et al. "Evaluation of a single surgeon's learning curve of laparoscopic pancreaticoduodenectomy: risk-adjusted cumulative summation analysis", Surgical Endoscopy, 35(6), pp. 2870-2878 (2021).

17. Safaei, A.S., Kazemzadeh, R.B., and Niaki, S.T.A. "Multi-objective economic statistical design of Xbar control chart considering Taguchi loss function", The International Journal of Advanced Manufacturing Technology, 59(9-12), pp. 1091-1101 (2012).

18. Liu, H.H., Song, Y.Y., and Yang, G.L. "Crossefficiency evaluation in data envelopment analysis based on prospect theory", European Journal of Operational Research, 273(1), pp. 364-375 (2019).

19. Chowdhury, H. and Zelenyuk, V. "Performance of hospital services in Ontario: DEA with truncated regression approach", Omega, 63, pp. 111-122 (2016).

20. Omrani, H., Keshavarz, M., and Ghaderi, S.F. "Evaluation of supply chain of a shipping company in Iran by a fuzzy relational network data envelopment analysis model", Scientia Iranica, Transactions E, Industrial Engineering, 25(2), pp. 868-890 (2018).

21. Sego, L.H., Reynolds, M.R., and Woodall, W.H. "Riskadjusted monitoring of survival times", Statistics in Medicine, 28(9), pp. 1386-1401 (2009).

22. Lawless, J.F., Statistical Models and Methods for Lifetime Data, New York: Wiley (2003). 
23. Asadzadeh, S. and Baghaei, A. "Robust AFT-based monitoring procedures for reliability data", Quality Technology \& Quantitative Management, 17(1), pp. 115 (2020).

24. Asadzadeh, S. and Aghaie, A. "Improving the product reliability in multistage manufacturing and service operations", Quality and Reliability Engineering International, 28(4), pp. 397-407 (2012).

25. Tzeng, G.H. and Huang, J.J., Fuzzy Multiple Objective Decision Making, Boca Raton, FL: Chapman and Hall/CRC (2013).

26. Deb, K., Pratap, A., Agarwal, S., et al. "A fast and elitist multiobjective genetic algorithm", IEEE Transactions on Evolutionary Computation, 6(2), pp. 182-197 (2002).

27. Madani, H., Arshadi Khamseh, A., and TavakkoliMoghaddam, R. "Solving a new bi-objective model for relief logistics in a humanitarian supply chain by bi-objective meta-heuristics in algorithms", Scientia Iranica, Transactions E, Industrial Engineering, 28(5), pp. 2948-2971 (2021).

28. Bernstein, A.D. and Parsonnet, V. "Bedside estimation of risk as an aid for decision-making in cardiac surgery", The Annals of Thoracic Surgery, 69(3), pp. 823-828 (2000).

\section{Biographies}

Navid Rafiei is currently a $\mathrm{PhD}$ candidate in Industrial Engineering at Islamic Azad University, North
Tehran Branch, Iran. His research interests include statistical quality control in healthcare, survival data analysis, multiple-criteria decision-making, and data envelopment analysis.

Shervin Asadzadeh is an Assistant Professor at Islamic Azad University, North Tehran Brach, Iran. He held his $\mathrm{PhD}$ in Industrial Engineering from Khajeh Nasir Toosi University of Technology in Iran (granted the honor of pursuing the PhD program). He has been teaching statistical methods, design of experiments, time series analysis, advanced statistical quality control, quality management and productivity, and engineering statistics at K.N. Toosi University of Technology, Allameh Tabataba'i University, and Islamic Azad University. He has published many papers in the area of statistical process control in high-quality international journals such as Quality and Reliability Engineering International, Quality Technology and Quantitative Management, Statistical Computation and Simulation, Communications in Statistics, and so on. He has been a member of National Elites Foundation of Iran since 2010 and he was selected as one of the researchers of the year in 2011 and 2017. His primary research interests include statistical quality control, reliability, survival data analysis, applied statistics in healthcare, robust statistics, and simulation. He is also a member of Iranian Institute of Industrial Engineering and Iranian Statistical Society. 